\title{
The Associations of Arcus senilis with Age and Metabolic Abnormalities
}

Steven B Leichter ${ }^{1 *}$, Jeff Johnson ${ }^{2}$, Michael Ammerman ${ }^{2}$ and Susan Egbert ${ }^{2}$

${ }^{1}$ The Southern Diabetes Foundation, Mercer University School of Medicine, Columbus, USA

${ }^{2}$ Vision Service Plan, Sacramento, USA

\begin{abstract}
From a voluntary ophthalmic screening program, involving 1876 people, carried out in Columbus, Georgia, $273(14.6 \%)$ were found to have arcus senilis. $973(51.9 \%)$ of the total population screened, were found to have evidence of a high risk of hypertension, hyperglycemia, or hyperlipidemia, and were invited to participate in further assessments. $623(64.0 \%)$ of these high risk subjects, including all of the patients with arcus senilis, agreed to further testing. $70 \%$ of the subjects had abnormal BMI, $64 \%$ had abnormal blood pressure, and $64 \%$ had evidence of abnormal fasting blood sugar, either by elevated fasting blood sugar or $\mathrm{Hgb} \mathrm{A} 1 \mathrm{c}>6 \%$. A minority had abnormality of LDL cholesterol, HDL cholesterol, or fasting serum triglycerides. Comparisons were then made between the 273 patients who had arcus senilis versus the 350 patients, who did not. Patients, who had arcus senilis has lower BMI values (32.6 $\left.\pm 0.4 \mathrm{vs} .34 .6 \pm 0.4 \mathrm{~kg} / \mathrm{m}^{2}, \mathrm{p}=0.005\right)$ and higher fasting blood sugar ( $133.6 \pm 3.6 \mathrm{vs} .125 .7 \pm 2.3 \mathrm{mg} /$ $\mathrm{dl}, p=0.0488)$ than those who did not have arcus. By multi-variate analysis, increasing age $(p<001)$, fasting blood sugar $(p<0.004), \mathrm{Hgb} \mathrm{A1c}(p<0.01)$, systolic $(p<0.007)$ and diastolic $(p=0.02)$ blood pressure, and decreasing BMI $(p=0.012)$ were associated with increasing prevalence of arcus senilis. Fasting LDL cholesterol, HDL cholesterol, LDL/HDL ratios, or serum triglycerides showed no association with the prevalence of arcus. We conclude that hyperglycemia and hypertension, but not hyperlipidemia, are the primary metabolic characteristics associated with the occurrence of arcus senilis, and age appears to have a significant association with the occurrence of arcus. These observations suggest that older individuals, who are found to have arcus senilis, should be screened for evidence of hyperglycemia.
\end{abstract}

Keywords: Arcus senilis; Hyperlipidemia; Hyperglycemia; Corneal arcus

\section{Introduction}

The clinical importance of arcus senilis is unclear. Arcus senilis (also termed corneal arcus) is an opaque ring around the outer portion of the cornea which has been associated with increased cardiac risk and hypercholesterolemia [1-4]. However, it has also been associated with diabetes, increasing age, alcoholism, hypertension and cigarette smoking [5]. One large population study disputed significance in the association of corneal arcus with cardiovascular disease, in contrast to xanthelasma and cardiovascular disease [6]. Both arcus and hypercholesterolemia are more prevalent with increasing age, but this is also true for other risks for cardiovascular disease, such as hypertension and hyperglycemia [3,5-8]. Since the prevalence of dyslipidemia, hyperglycemia and hypertension increases with age, there is no data available to assess which of these conditions is more strongly associated with arcus senilis.

As part of an eye care screening program in Columbus, Georgia on 623 subjects, we identified 273 patients, who had arcus senilis. We carried out metabolic assessments for hyperlipidemia, hypertension and hyperglycemia in these subjects, and compared these results to a comparable group of 350 patients, who participated in the program, but who did not have arcus senilis. This provided us with the opportunity to determine which, if any of these conditions were more strongly associated with the presence of arcus senilis versus their prevalence and severity in an otherwise similar population of control subjects.

\section{Methods}

This study was carried out between 2007 and 2008. All subjects were voluntary participants in a free ophthalmic screening program in Columbus, Georgia, sponsored by Vision Service Plan (VSP). Every patient in the program signed a consent form, consistent with requirements for participation in a screening program, as defined by regulations in the State of Georgia. As part of the screening, patients received an ophthalmic examination, which included observation of the presence or absence of any degree of arcus senilis. In screening for arcus senilis, patients were assessed for the presence or absence of arcus.

In addition to the ophthalmic examination, patients provided a brief medical history and had measurement of body mass index and blood pressure. The medical history included whether subjects had a known diagnosis of diabetes, hypertension, hyperlipidemia, or a family history of these disorders. Patients, who had a history or family history of one or more of these disorders, or were found to have arcus, or had abnormal body mass index (BMI), or blood pressure were referred to the Columbus Research Foundation for further evaluation.

Those patients, who were referred, had additional laboratory tests obtained at no charge. These tests included: fasting serum glucose; fasting lipid profile; and hemoglobin A1c. All testing was carried out by point-of-service technology, following a 12 hour fast. Fasting blood sugar was measured by the Accu-Chek Aviva, (Roche Diagnostics, 9115 Hague Rd., Indianapolis, IN 46256. Hemoglobin A1c was measured by the A1c Now, (Bayer Healthcare, 510 Oakmead Pkwy, Sunnyvale, CA 94085) Fasting lipid profile was measured by the Cardio Chek P.A,

*Corresponding author: Steven B Leichter, Clinical Professor of Internal Medicine, Mercer University School of Medicine, Columbus, GA, USA, E-mail: Sugardoc6@aol.com

Received July 05, 2013; Accepted September 23, 2013; Published September 28, 2013

Citation: Leichter SB, Johnson J, Ammerman M, Egbert S (2013) The Associations of Arcus senilis with Age and Metabolic Abnormalities. J Diabetes Metab 4: 293 doi:10.4172/2155-6156.1000293

Copyright: (C) 2013Leichter SB, et al. This is an open-access article distributed under the terms of the Creative Commons Attribution License, which permits unrestricted use, distribution, and reproduction in any medium, provided the original author and source are credited. 


\begin{tabular}{|l|c|c|c|}
\hline Observation & $\begin{array}{c}\text { \# Males (\% of } \\
\mathbf{2 0 7})\end{array}$ & $\begin{array}{c}\text { \# Females } \\
\mathbf{4 1 6} \text { of }\end{array}$ & $\begin{array}{c}\text { Total (\% of 623 } \\
\text { Ss) }\end{array}$ \\
\hline BMI & $131(63 \%)$ & $304(73 \%)$ & $435(70 \%)$ \\
\hline Blood Pressure & $149(72 \%)$ & $252(61 \%)$ & $401(64 \%)$ \\
\hline Fasting Blood Sugar & $143(69 \%)$ & $253(61 \%)$ & $396(64 \%)$ \\
\hline Hemoglobin A1c & $86(42 \%)$ & $184(44 \%)$ & $270(43 \%)$ \\
\hline LDL-cholesterol & $70(34 \%)$ & $153(37 \%)$ & $223(36 \%)$ \\
\hline HDL-cholesterol & $151(73 \%)$ & $296(71 \%)$ & $447(72 \%)$ \\
\hline Serum triglycerides & $49(24 \%)$ & $119(29 \%)$ & $168(27 \%)$ \\
\hline
\end{tabular}

Table 1: Prevalence of Metabolic Abnormalities in the Entire Group of 623 Patients studied in detail.

\begin{tabular}{|c|c|c|c|}
\hline Observation & \# Males (\% of 207) & $\begin{array}{c}\text { \# Females } \mathbf{4} \% \text { of } \\
\mathbf{4 1 6}\end{array}$ & $\begin{array}{c}\text { Total (\% of 623 } \\
\text { Ss) }\end{array}$ \\
\hline BMI & $131(63 \%)$ & $304(73 \%)$ & $435(70 \%)$ \\
\hline Blood Pressure & $149(72 \%)$ & $252(61 \%)$ & $401(64 \%)$ \\
\hline Fasting Blood Sugar & $143(69 \%)$ & $253(61 \%)$ & $396(64 \%)$ \\
\hline Hemoglobin A1c & $86(42 \%)$ & $184(44 \%)$ & $270(43 \%)$ \\
\hline LDL-cholesterol & $70(34 \%)$ & $153(37 \%)$ & $223(36 \%)$ \\
\hline HDL-cholesterol & $151(73 \%)$ & $296(71 \%)$ & $447(72 \%)$ \\
\hline Serum triglycerides & $49(24 \%)$ & $119(29 \%)$ & $168(27 \%)$ \\
\hline
\end{tabular}

Table 2: Comparison of prevalence of abnormality for patients with arcus versus those without arcus

\begin{tabular}{|c|c|c|c|c|}
\hline Parameter & Ss with Arcus & Ss without Arcus & T-test & $\mathbf{p}$ \\
\hline Age (Yrs) & $57.95 \pm 0.46$ & $50.1 \pm 0.8$ & 5.5938 & $<0.001$ \\
\hline BMI & $32.6 \pm 0.4$ & $34.6 \pm 0.4$ & 3.4818 & $=0.0005$ \\
\hline Systolic BP & $135.2 \pm 1.1$ & $136.8 \pm 1.0$ & 1.0644 & $=0.2875$ \\
\hline Diastolic BP & $83.6 \pm 0.8$ & $83.5 \pm 0.6$ & 0.0810 & $=0.9355$ \\
\hline Hemoglobin A1c (\%) & $5.7 \pm 0.1$ & $5.6 \pm 0.1$ & 0.6964 & $=0.4865$ \\
\hline $\begin{array}{c}\text { Fasting Serum Glucose } \\
\text { (mg/dl) }\end{array}$ & $133.6 \pm 3.6$ & $125.7 \pm 2.3$ & 1.9739 & $=0.0488$ \\
\hline $\begin{array}{c}\text { LDL-cholesterol (mg/dl) } \\
\text { HDL -cholesteol (mg/dl) }\end{array}$ & $112 \pm 2.9$ & $107 \pm 2.5$ & 1.1835 & $=0.2371$ \\
\hline $\begin{array}{c}\text { Serum Triglycerides } \\
\text { (mg/dl) }\end{array}$ & $121.5 \pm 5.5$ & $129.4 \pm 5.7$ & 0.9798 & $=0.3276$ \\
\hline
\end{tabular}

Table 3: Comparison of age, sex, BMI, blood pressure, fasting blood sugar, and lipid levels in patients with and without arcus senilis.

(Polymer Technology Systems (PTS), 7736 Zionsville Rd, Indianapolis, IN, 46268). The results were analyzed by Systat and SAS software. Multivariate analyses were carried out, using SAS Software to correct for the influence of age, among other factors.

Normal values for lipids, blood pressure, and blood sugar were defined by ATP III criteria [9]. Abnormal hemoglobin A1c levels were based on the criteria of the American Diabetes Association for clinically significant abnormality of hemoglobin A1c of $6 \%$ or greater [10-12]. Body mass index was calculated by NIH criteria [13]. Abnormal body mass index was defined as $>30$, according to these criteria.

\section{Results}

A total of 1876 people had the initial, complete ophthalmic examination. $659(35 \%)$ were male and $1217(65 \%)$ were female. Arcus senilis was observed in $273(14.6 \%)$ of these patients. Of the original group of subjects, 973 (51.9\%) were identified as having a risk of metabolic disease by the above criteria or having arcus senilis by examination. 623 (64.0\%) of these patients, including all of the patients with arcus senilis, participated in further assessments. There were no significant differences in any of the characteristics studies, including age or sex distribution, between the participants, who elected to complete further evaluations and those, who did not (data not shown). A similar distribution of men and women existed in the group with arcus as the entire study population: 98 of the patients with arcus (36\%) were men and 175 (64\%) were women (pnsvs the sex distribution for the entire population). In addition, the sex distribution in the group that had corneal arcus was similar to the group of 350 patients, studied in detail, which did not have arcus senilis ( $36 \%$ men vs. $31 \%$ men) (p ns).

The population of patients with corneal arcus was distinctly older than the original group of 1876 people, or the comparator group, studied in detail of 350 subjects. Patients found to have arcus senilis had a mean age of $57.95 \pm .46$ years versus a mean age of $48.1 \pm 0.4$ years in the original group $(\mathrm{p}<0.01)$. The 98 male patients found to have arcus senilis were $39-79$ years of age (mean $57.14 \pm .95 \mathrm{yrs}$ ). The 175 female patients found to have arcus senilis were 26-84 years of age (mean $58.4 \pm .83$ years) ( $\mathrm{p} \mathrm{ns}$ ). Similarly, the patients with corneal arcus were significantly older than the mean age $(50.1 \pm 0.8$ years) of the patients in the comparator group that was studied in detail $(\mathrm{p}<0.001)$. This was true for men with corneal arcus $(57.14 \pm 1.05$ years) versus those without $(49.2 \pm 1.55$ years $)(\mathrm{p}<0.001)$, and women with corneal arcus ( $58.4 \pm 0.83$ years) versus those without $(50.5 \pm 0.97$ years $)(p<0.002)$.

Metabolic abnormalities were common in the entire group of 623 patients who presented to CRF for complete assessment (Table 1). The majority had abnormal BMI, blood pressure, HDL-cholesterol and blood sugar. A large minority also had abnormal hemoglobin Alc levels. However, elevated LDL was found in only $36 \%$ and $27 \%$ had a serum triglyceride level $>150 \mathrm{mg} / \mathrm{dl}$. There was no difference in the prevalence of these findings, when analyzed by gender.

Comparison of the group of subjects with corneal arcus to those without demonstrated that the comparator group was similar in all respects to the group with arcus senilis, except for age, as noted above. Sex distribution was the same. No difference was observed in the prevalence of obesity, hypertension, abnormal lipid fractions, or hyperglycemia in the patients who had corneal arcus versus those who did not (Table 2). The prevalence of abnormal blood sugar metabolism was assessed both by fasting blood sugar and hemoglobin A1c levels. The lipid fractions studied included LDL-cholesterol, HDL cholesterol, and fasting serum triglycerides.

The levels of each of these characteristics were compared in the sub-group of patients with corneal arcus versus those who did not have corneal arcus (Table 3). As noted above, the patients, who had arcus, were significantly older $(\mathrm{p}<0.01)$. They had a significantly higher fasting blood sugar $(\mathrm{p}=0.0488)$, although there was no difference in the hemoglobin Alc observed in each group. The patients without arcus had a significantly lower HDL-cholesterol level $(p=0.0335)$ and a significantly greater BMI $(\mathrm{p}=0.005)$. There was no difference observed in LDL-cholesterol, blood pressure or serum triglycerides. The ratio between HDL and LDL cholesterol was similar between both groups.

An age-adjusted analysis demonstrated the increasing occurrence of arcus senilis with increasing age, although not all of the 273 subjects could be included in the analysis, due to uncertainty about birthdates in a small minority (Table 4). Multivariate regression analysis was carried out on these observations. It demonstrated that the primary variables, associated with corneal arcus in this population were increasing age $(p<0.0001)$, increasing fasting blood sugar $(p=0.0037)$, increasing HgbA1c $(\mathrm{p}=0.01)$, increasing systolic blood pressure $(\mathrm{p}=0.0068)$ and diastolic blood pressure $(\mathrm{p}=0.02)$, and decreasing BMI $(\mathrm{p}=0.012)$. There was no significant association with LDL-cholesterol $(\mathrm{p}=0.3455)$, HDL $(\mathrm{p}=0.2311)$, serum triglycerides $(0.3436)$ or raw body weight $(\mathrm{p}=0.642)$. When analyzed by metabolic characteristics and gender, these same relationships were found for age, BMI, blood pressure, and fasting blood sugar ( $\mathrm{p}<0.05$ for each). 


\begin{tabular}{|c|c|c|c|}
\hline Age & With Arcus & Without Arcus & Percent with Arcus \\
\hline $0-9$ & 0 & 0 & $0 \%$ \\
\hline $10-19$ & 0 & 9 & $0 \%$ \\
\hline $20-29$ & 4 & 35 & $10 \%$ \\
\hline $30-39$ & 9 & 55 & $14 \%$ \\
\hline $40-49$ & 61 & 70 & $47 \%$ \\
\hline $50-59$ & 100 & 102 & $50 \%$ \\
\hline $60-69$ & 57 & 58 & $50 \%$ \\
\hline $70-$-above & 17 & 21 & $45 \%$ \\
\hline
\end{tabular}

Table 4: Occurrence of arcus senilis by age.

\section{Discussion}

Arcus senilis is thought to be a marker for increased risk of cardiovascular disease [4] and it is thought to be associated with lipid abnormalities [14]. However, while the literature seems to support the association with cardiovascular risk with some consistency, it is unclear about relationships between arcus and specific metabolic abnormalities. Associations between the occurrence of corneal arcus and lipid deposition in other tissues such as aortic atherosclerosis and Achilles tendonous xanthomas have been shown [2]. One South African study demonstrated association of corneal arcus with abnormalities of LDL and HDL cholesterol levels [15]. However, the study did not factor in the effects of age. On the other hand, Chen et al. [16] noted that the associations between the occurrence of arcus and cardiovascular risk were abolished, when adjusted for age.

The present study looked at the prevalence of hyperlipidemia, hypertension, abnormalities in BMI, and hyperglycemia in two closely matched groups: patients, who were recruited for free eye exams and found to have arcus senilis; and patients without arcus senilis recruited from the same group, who by BMI, blood pressure measurement or personal or family history were thought to have increased risk of one or more of these metabolic conditions. The results included age-adjusted, multivariate analyses, and they demonstrate a primary relationship between increasing age and the occurrence of arcus senilis. They also suggest a relationship among hyperglycemia, hypertension and corneal arcus, and decreasing BMI and arcus. In contrast, the results clearly show no direct association between lipid levels and corneal arcus, most especially LDL-cholesterol levels, when corrected for age.

That age is an important association with the occurrence of arcus senilis is well-recognized in the literature $[3,5-8,17,18]$. However, that hyperglycemia is, perhaps, the most important metabolic abnormality to be significantly associated with this condition is not. At least two prior publications have reported associations between hyperglycemia and the occurrence of arcus senilis [19,20], while one did not confirm this association [16]. However, the negative study was carried out in an unusually thin, rural Indian population, which may, in part, explain its findings. The present study emphasizes the significance of the association in an American population and, perhaps suggests more details about it. This is because in the present study, the prevalence of arcus was significantly associated with hyperglycemia and hypertension. This raises the question of whether hyperglycemia alone or in combination with hypertension is a contributing factor to the occurrence of arcus. The inverse relationship between arcus and BMI in this population suggests the importance of hyperglycemia and hypertension specifically in the relationship with the occurrence of arcus. Whatever the primary causal relationship among these factors and arcus senilis is, this and the previous studies in the literature, suggest that patients, who are found to have arcus senilis, should be screened for hyperglycemia. That arcus would be an indicator to suggest the presence of some degree of clinically significant hyperglycemia is an important, new consideration.

\section{References}

1. Hoogerbrugge $N$, Happee $C$, van Domburg R, Poldermans D, van den Brand MJ (1999) Corneal arcus: indicator for severity of coronary atherosclerosis? Neth J Med 55: 184-187.

2. Zech LA Jr, Hoeg JM (2008) Correlating corneal arcus with atherosclerosis in familial hypercholesterolemia. Lipids Health Dis 7: 7 .

3. Fernández A, Sorokin A, Thompson PD (2007) Corneal arcus as coronary artery disease risk factor. Atherosclerosis 193: 235-240.

4. Ang M, Wong W, Park J, Wu R, Lavanya R, et al. (2011) Corneal arcus is a sign of cardiovascular disease, even in low-risk persons. Am J Ophthalmol 152 864-871.

5. Harris MI, Hadden WC, Knowler WC, Bennett PH (1987) Prevalence of diabetes and impaired glucose tolerance and plasma glucose levels in U.S. population aged 20-74 yr. Diabetes 36: 523-534.

6. Christoffersen M, Frikke-Schmidt R, Schnohr P, Jensen GB, Nordestgaard BG et al. (2011) Xanthelasmata, arcus corneae, and ischaemic vascular disease and death in general population: prospective cohort study. BMJ 343: d5497.

7. Assmann G, Schulte H (1988) The Prospective Cardiovascular Münster (PROCAM) study: prevalence of hyperlipidemia in persons with hypertension and/or diabetes mellitus and the relationship to coronary heart disease. Am Heart J 116: 1713-1724.

8. Poulsen MK, Henriksen JE, Vach W, Dahl J, Møller JE, et al. (2010) Identification of asymptomatic type 2 diabetes mellitus patients with a low, intermediate and high risk of ischaemic heart disease: is there an algorithm? Diabetologia 53 659-667.

9. National Heart Lung and Blood Institute (2004) Third Report of the Expert Panel on the Detection, Evaluation and Treatment of High Blood Cholesterol in Adults (Adult Treatment Panel III)

10. American Diabetes Association (2010) Standards of medical care in diabetes--2010. Diabetes Care 33: S11-61.

11. (2009) International Expert Committee Report on the Role of the A1c Assay in the Diagnosis of Diabetes. Diabetes Care 32: 1327-1334.

12. American Diabetes Association (2012) Executive summary: Standards of medical care in diabetes--2012. Diabetes Care 35: S4-4S10.

13. National Heart, Lung, and Blood Institute (1998) Clinical Guidelines on the Identification, Evaluation, and Treatment of Overweight and Obesity in Adults: The Evidence Report. National Institutes of Health.

14. Rifkind BM (1972) Corneal arcus and hyperlipoproteinaemia. Surv Ophthalmo 16: $295-304$

15. Meyer D, Liebenberg PH, Maritz FJ (2004) Serum lipid parameters and the prevalence of corneal arcus in a dyslipidaemic patient population. Cardiovasc J S Afr 15: 166-169.

16. Chen HT, Chen HC, Hsiao CH, Ma DH, Chen YT, et al. (2009) Corneal arcus and cardiovascular risk factors in middle-aged subjects in Taiwan. Am J Med Sci 338: 268-272.

17. Fernandez AB, Keyes MJ, Pencina M, D'Agostino R, O'Donnell CJ, et al. (2009) Relation of corneal arcus to cardiovascular disease (from the Framingham Heart Study data set). Am J Cardiol 103: 64-66.

18. Vurgese S, Panda-Jonas S, Saini N, Sinha A, Nangia V, et al. (2011) Cornea arcus and its associations with ocular and general parameters: the Central India Eye and Medical Study. Invest Ophthalmol Vis Sci 52: 9636-9643.

19. Chua BE, Mitchell P, Wang JJ, Rochtchina E (2004) Corneal arcus and hyperlipidemia: findings from an older population. Am J Ophthalmol 137: 363365.

20. Moss SE, Klein R, Klein BE (2000) Arcus senilis and mortality in a population with diabetes. Am J Ophthalmol 129: 676-678. 